\title{
TITLE:
}

\section{Integration of GaN with Si using a AuGe-mediated wafer bonding technique}

\author{
$\operatorname{AUTHOR}(\mathrm{S}):$
}

Funato, M; Fujita, S; Fujita, S

\section{CITATION:}

Funato, M ... [et al]. Integration of GaN with Si using a AuGe-mediated wafer bonding technique. APPLIED PHYSICS LETTERS 2000, 77(24): 3959-3961

ISSUE DATE:

2000-12-11

URL:

http://hdl.handle.net/2433/39640

\section{RIGHT:}

Copyright 2000 American Institute of Physics. This article may be downloaded for personal use only. Any other use requires prior permission of the author and the American Institute of Physics. 


\title{
Controlling the noncentrosymmetry of azodye-doped polymers by nonresonant dual-frequency coherent excitation
}

\author{
Jinhai $\mathrm{Si}^{\mathrm{a})}$ and Jianrong Qiu \\ Photon Craft Project, ICORP, JST, Keihanna-Plaza, Super-Lab. 2-5, 1-7 Hikaridai, Seika-cho, \\ Kyoto 619-0237, Japan \\ Kazuyuki Hirao \\ Division of Material Chemistry, Faculty of Engineering, Kyoto University, Sakyo-ku Kyoto 606-8501, Japan
}

(Received 3 August 2000; accepted for publication 16 October 2000)

\begin{abstract}
All-optical control of the noncentrosymmetry of azodye-doped polymer films was demonstrated by nonresonant dual-frequency coherent excitation. The tensor analysis of the photoinduced noncentrosymmetry was performed by monitoring second harmonic generation (SHG) of the samples. The quadratic dependence of SHG on the film thickness showed that a $\chi^{(2)}$ grating satisfying the phase-matching condition for SHG was optically induced in the polymer films, indicating this nonresonant all-optical poling can be used to tailor the symmetry of bulk samples.

(C) 2000 American Institute of Physics. [S0003-6951(00)01851-9]
\end{abstract}

Controlling and manipulating the external degrees of freedom of atoms and molecules are a central subject in modern physics and chemistry. In studies of condensed matter, scanning probe microscopes enable selection and manipulation of individual atoms and molecules. ${ }^{1}$ In chemistry researchers have aimed at control of the spatial orientation of molecules because it allows for studies of orientational effects in chemical reactions. ${ }^{2}$ The control can be achieved by applying a strong static electric field for molecules possessing permanent dipole moments, ${ }^{3}$ or by introducing a strong laser field for neutral molecules. ${ }^{4,5}$

Dual-frequency coherent laser excitation at fundamental and second harmonic frequencies has been shown to permit the achievement of large polar orientation efficiency in azodye-doped polymers. ${ }^{6}$ One breakthrough with such an alloptical poling technique is that it leads to an automatic molecular organization with a period satisfying the phasematching conditions for second harmonic generation (SHG). Additionally, using appropriate combinations of the writing beam polarizations, dual-frequency coherent excitation enables full control of the molecular polar order, which permit complete all-optical tailoring of the symmetry of polymer materials. ${ }^{7}$ The physical mechanism of all-optical poling consists of two processes: the orientational hole burning and the reorientation of azodye molecules. ${ }^{8,9}$ Under the phasecoherent excitation of a fundamental light along with its second-harmonic light, an orientational hole burning of azodye molecules occurs through the interference of the twophoton absorption at the fundamental frequency and the onephoton absorption at the doubling frequency. The orientational hole-burning is followed by a reverse trans-cis-trans isomerization, which finally leads to a net permanent polar orientation of molecules. This technique possesses some advantages as follows: phase matching for SHG can automatically be achieved, and a micropatterning of the molecular

${ }^{a)}$ Electronic mail: jhsi@photon.jst.go.jp polar orientation can be simply achieved by scanning the focal area in the polymer sample.

In a resonant optical excitation process, however, it appears difficult to control the symmetry of bulk samples using this all-optical poling owing to the material absorption and it is also difficult for the SHG conversion efficiency to benefit from phase matching achieved automatically in large propagation distances. ${ }^{10}$ Recently, we successfully achieved alloptical poling of azodye-doped polymethylmethacrylate (PMMA) films using the $1500 \mathrm{~nm}$ fundamental and the 750 $\mathrm{nm}$ second-harmonic light of a femtosecond laser. ${ }^{11} \mathrm{Al}-$ though the SHG conversion efficiency still was low due to the use of thin films of only several micrometers, the nonresonant excitation should permit the use of larger film thickness, which should in turn result in a significant increase of the conversion efficiency. In this letter, we experimentally demonstrated all-optical control of the symmetry of azodyedoped PMMA thick films by nonresonant dual-frequency coherent excitation. During the writing process, samples were irradiated simultaneously by the coherent superposition of the $1300 \mathrm{~nm}$ fundamental and the $650-\mathrm{nm}$ second-harmonic light of a femtosecond laser. The characteristic kinetics of the photoinduced molecular polar orientation and the tensor analysis were performed by monitoring SHG of the samples. The quadratic dependence of SHG on the film thickness showed that a $\chi^{(2)}$ grating satisfying the phase-matching condition for SHG was optically induced in the polymer films, indicating this nonresonant all-optical poling can be used to tailor the symmetry of bulk samples.

Samples in this study were prepared by dissolving to a $15 \%$ mass ratio purified disperse red 1 (DR1) and medium molecular weight PMMA in tetrahydorofuran. Films were spin deposited onto glass substrates, and dried for several hours at $160^{\circ} \mathrm{C}$. Films with different thickness were prepared by adjusting the viscosity of the solution and the revolutions per minute of the spin coater. According to the absorption spectra of the films, we know that the absorption band peaks at $480 \mathrm{~nm}$, and the film sample is almost transparent at the wavelength range of larger than $650 \mathrm{~nm}$. 


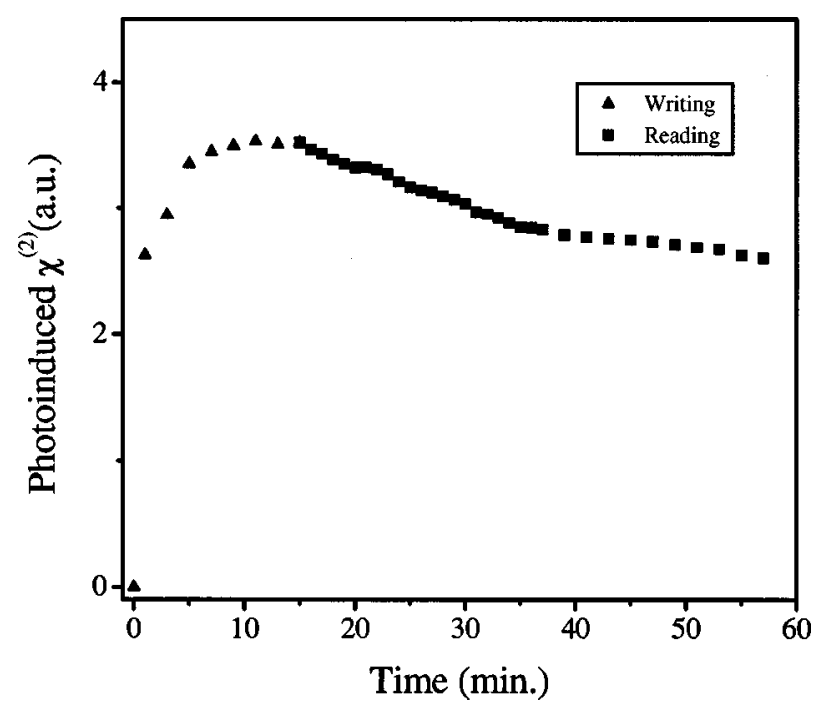

FIG. 1. Growth and decay processes of the photoinduced $\chi^{(2)}$ of a $73 \mu \mathrm{m}$ film.

A Ti:sapphire laser system with an optical parametric amplifier (OPA) was used for all-optical poling of the films, which emitted 150-fs-laser pulses at a repetition rate of 1 $\mathrm{kHz}$, and the wavelength of the laser from OPA could be tuned from 300 to $3000 \mathrm{~nm}$. The average power of the laser was about $50 \mathrm{~mW}$ at wavelengths ranging from 1300 to 1500 $\mathrm{nm}$. Writing beam $\omega$ for all-optical poling was split from the source beam and passed through a time-delay device and a $\lambda / 2$ plate to control the path length and the polarization of the beam, respectively. Another beam separated from the source beam was frequency doubled by a KDP crystal, served as another writing beam, and is denoted seed beam $2 \omega$. The two collinear writing beams were introduced into the film sample through a 6-cm-focal-length quartz lens. We achieved time superposition of pulses between the two writing beams by adjusting the time-delay device and observing the optical Kerr effect of $\mathrm{CS}_{2}$. During the all-optical poling process, the two writing beams were introduced simultaneously into the film sample; during the probe, beam $2 \omega$ was blocked by a shutter and only the beam $\omega$ remained incident.

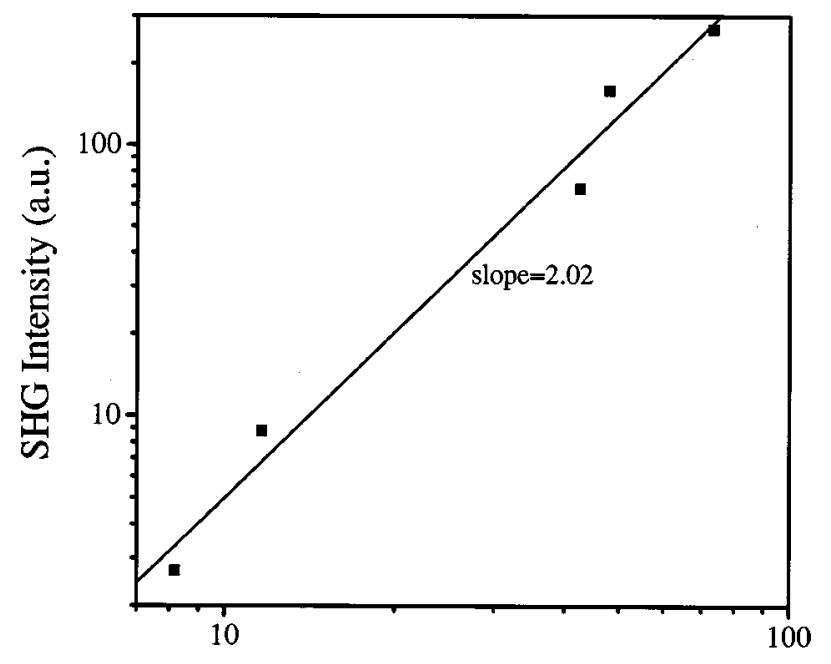

Film Thickness $(\mu \mathrm{m})$

FIG. 2. Log-log plots with a linear fit of slope near 2, displaying SHG intensity dependence on the film thickness squared. intensity dependence on the film thickness squared.
Downloaded 30 May 2007 to 130.54 .110 .22 . Redistribution subject to AIP license or copyright, see http://apl.aip.org/apl/copyright.jsp
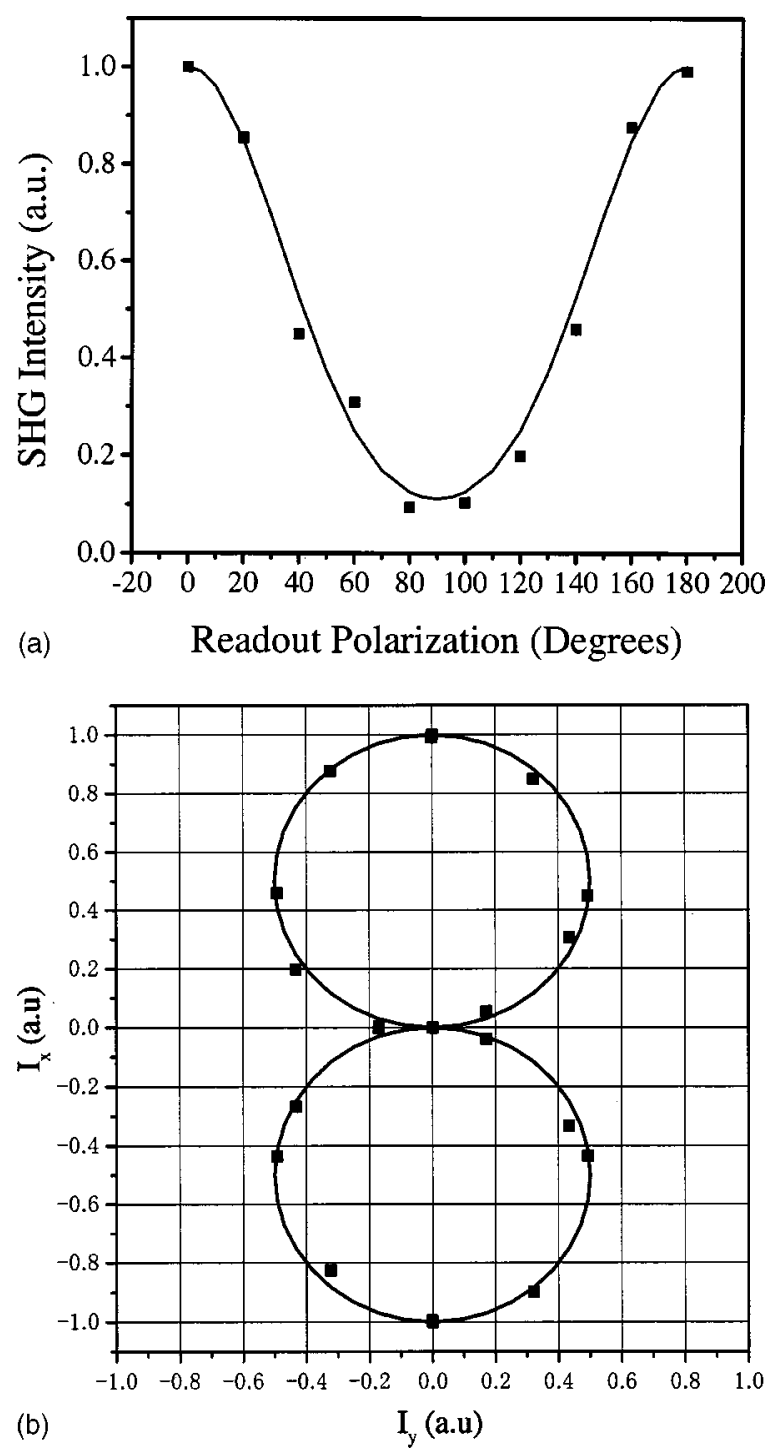

FIG. 3. Photoinduced $\chi^{(2)}$ symmetry of a $73 \mu \mathrm{m}$ film by nonresonant dualfrequency coherent excitation. (a): tensor data and fits for the $x$ component of SHG signals from the photoinduced $\chi^{(2)}$; (b): the data were obtained by simultaneous rotation of the input-beam polarization and the analyzing polarizer.

The SHG signal of beam $\omega$ was detected by a photomultiplier and observed and averaged by an oscilloscope. Beam $\omega$ passing through the sample was blocked by a heat-absorbing filter placed behind the sample, and allows only the SHG signal to pass through. Typical fluences of the two writing beams at the sample were $\sim 1.5 \mathrm{~mJ} / \mathrm{cm}^{2}$ for beam $\omega$ and $0.03 \mathrm{~mJ} / \mathrm{cm}^{2}$ for beam $2 \omega$, respectively.

By adjusting the delay device, we measured the dependence of photoinduced SHG on the temporal correlation of the two writing beams, and observed that all-optical poling of the films could have been performed only when the two writing beams were at a coherent superposition. This indicated that all-optical poling resulted from the coherent excitation of the two writing lights for azodye molecules in the films. Figure 1 shows the growth and decay processes of photoinduced $\chi^{(2)}$ of a film of thickness of $73 \mu \mathrm{m}$. It can be found that the photoinduced $\chi^{(2)}$ reaches its saturation value in $10 \mathrm{~min}$ for the condition described above.

Figure 2 shows the dependence of the SHG signal versus 
made after the photoinduced $\chi^{(2)}$ of the films increased into their saturation values. It can be found from the figure that as expected the SHG signal increases proportional to the square of the film thickness. A peak conversion efficiency of $0.3 \%$ was observed for the $73 \mu \mathrm{m}$ film and a fundamental light intensity of $9 \mathrm{GW} / \mathrm{cm}^{2}$ incident on the sample. Obviously, this high conversion efficiency benefited from quasi-phasematched SHG in the thick film. We also observed that SHG intensities of the films departed from the quadratic length dependence when thickness of the films was larger than 90 $\mu \mathrm{m}$. This was due to the group velocity mismatch between the writing beam $\omega$ and the writing beam $2 \omega$.

For tensor analysis, we performed experiments to measure the $x$ and the $y$ components of the SHG signals by keeping the back polarizer fixed in the vertical $(x)$ or horizontal $(y)$ position and rotating the readout-beam polarization. ${ }^{6}$ Figure 3(a) displays the tensor curve for the $x$ component for a $73 \mu \mathrm{m}$ film written by the 1300- and 650$\mathrm{nm}$-seed beams. The tensor curve was fitted by the general polarization expressions as the following, ${ }^{12}$

$$
\begin{aligned}
& \left|E_{x}^{2 \omega}\right|^{2} \propto\left|\chi_{x x x}^{(2)} \cos ^{2} \theta+2 \chi_{x x y}^{(2)} \cos \theta \sin \theta+\chi_{x y y}^{(2)} \sin ^{2} \theta\right|^{2}, \\
& \left|E_{y}^{2 \omega}\right|^{2} \propto\left|\chi_{y y y}^{(2)} \sin ^{2} \theta+2 \chi_{y y x}^{(2)} \cos \theta \sin \theta+\chi_{y x x}^{(2)} \cos ^{2} \theta\right|^{2}
\end{aligned}
$$

where $\theta$ is the readout beam polarization measured with respect to the vertical $x$ axis, and the best fits gave

$$
\chi_{x x x}^{(2)} / \chi_{x y y}^{(2)} \approx 3, \text { and } \chi_{x x y}^{(2)}=0 .
$$

From the similar measurements for the y component, we obtained

$$
\left.\chi_{x x x}^{(2)} / \chi_{y y y}^{(2)}\right\rangle 12 \text {, and } \chi_{y y x}^{(2)}=0 .
$$

Figure 3(b) shows another result measured for the photoinduced $\chi^{(2)}$ symmetry of the film, where $I_{x}$ and $I_{y}$ respresent the $x$ and the $y$ components of the SHG signals, respectively. ${ }^{6}$ The data were obtained by simultaneous rota- tion of the input-beam polarization and the analyzing polarizer. These tensor analyses indicate that for collinearly polarized writing beams, the photoinduced $\chi^{(2)}$ in the film exhibited axial symmetry along the writing beam polarization direction.

To compare SHG intensities of the films with that of a Y-cut quartz of thickness of $1 \mathrm{~mm}$, we also measured the SHG intensity ratio for the $73 \mu \mathrm{m}$ film, $I_{s} / I_{q}$, where $I_{s}$ and $I_{q}$ are the SHG intensities of the film and a Y-cut quartz for the same readout power, respectively. The SHG intensity ratio between the film and the quartz, $I_{s} / I_{q}$, was measured to be 43.6 .

In summary, we experimentally demonstrated all-optical control of the noncentrosymmetry of azodye-doped PMMA films by nonresonant dual-frequency coherent excitation. The tensor analysis of the photoinduced noncentrosymmetry was performed. The quadratic dependence of SHG on the film thickness showed this nonresonant all-optical poling can be used to tailor the symmetry of bulk samples.

${ }^{1}$ J. A. Stroscio and D. M. Eigler, Science 254, 1319 (1991).

${ }^{2}$ P. Brumer and M. Shapiro, Annu. Rev. Phys. Chem. 43, 257 (1992).

${ }^{3}$ B. Friedrich and D. R. Herschbach, Nature (London) 353, 412 (1991).

${ }^{4}$ H. Sakai, C. P. Safvan, J. J. Larsen, K. M. Hilligsoe, K. Hald, and H. Stapelfeldt, J. Chem. Phys. 110, 10235 (1999).

${ }^{5}$ D. M. Villeneuve, S. A. Aseyev, P. Dietrich, M. Spanner, M. Yu. Ivanov, and P. B. Corkum, Phys. Rev. Lett. 85, 542 (2000).

${ }^{6}$ C. Fiorini, F. Charra, J.-M. Nunzi, and P. Raimond, J. Opt. Soc. Am. B 14, 1984 (1997).

${ }^{7}$ A. Etile, C. Fiorini, F. Charra, and J.-M. Nunzi, Phys. Rev. A 56, 3888 (1997).

${ }^{8}$ F. Charra, F. Kajzar, J.-M. Nunzi, P. Raimond, and E. Idiart, Opt. Lett. 18, 941 (1993).

${ }^{9}$ F. Charra, F. Devaux, J.-M. Nunzi, and P. Raimond, Phys. Rev. Lett. 68, 2440 (1992).

${ }^{10}$ J. Si, K. Kitaoka, T. Mitsuyu, P. Ye, and K. Hirao, J. Appl. Phys. 85, 8018 (1999).

${ }^{11}$ K. Kitaoka, J. Si, T. Mitsuyu, and K. Hirao, Appl. Phys. Lett. 75, 157 (1999).

${ }^{12}$ T. J. Driscoll and N. M. Lawandy, J. Opt. Soc. Am. B 11, 355 (1994). 